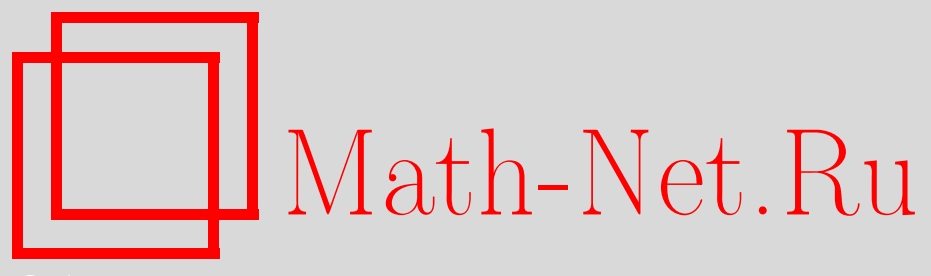

Ю. В. Третьяченко, В. В. Чистяков, Принцип выбора для поточечно ограниченных последовательностей функций, Матем. заметки, 2008, том 84, выпуск 3, 428439

DOI: https://doi.org/10.4213/mzm4230

Использование Общероссийского математического портала Math-Net.Ru подразумевает, что вы прочитали и согласны с пользовательским соглашением http: //www . mathnet.ru/rus/agreement

Параметры загрузки:

IP: 54.197 .130 .99

26 апреля 2023 г., 10:10:07

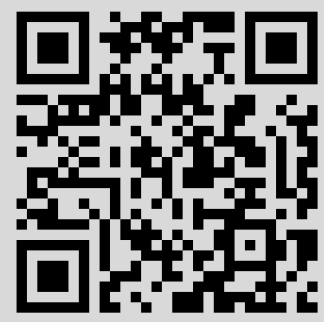


Том 84 выпуск 3 сентябрь 2008

УДК $517.27+517.544 .4$

\section{Принцип выбора для поточечно ограниченных последовательностей функций}

\section{Ю. В. Третьяченко, В. В. Чистяков}

Для числа $\varepsilon>0$ и вещественной функции $f$ на отрезке $[a, b]$ обозначим через $N(\varepsilon, f,[a, b])$ супремум множества тех номеров $n$, для которых в $[a, b]$ существует набор неналегающих отрезков $\left[a_{i}, b_{i}\right], i=1, \ldots, n$, таких, что $\left|f\left(a_{i}\right)-f\left(b_{i}\right)\right|>\varepsilon$ для всех $i=1, \ldots, n(\sup \varnothing=0)$. Доказана следующая теорема: если $\left\{f_{j}\right\}-n o-$ точечно ограниченная последовательность вещественных функиий на отрезке $[a, b]$ mакал, что $n(\varepsilon) \equiv \lim \sup _{j \rightarrow \infty} N\left(\varepsilon, f_{j},[a, b]\right)<\infty$ для любого $\varepsilon>0, \operatorname{mo}\left\{f_{j}\right\}$ содержит подпоследовательность, которая всюду на $[a, b]$ сходится к некоторой функции $f$ такой, что $N(\varepsilon, f,[a, b]) \leqslant n(\varepsilon)$ при любом $\varepsilon>0$. Показано, что основное условие в этой теореме, связанное с верхним пределом, необходимо для равномерно сходящейся последовательности $\left\{f_{j}\right\}$ и "почти" необходимо для всюду сходящейся последовательности измеримых функций и что многие поточечные принципы выбора, обобщающие классическую теорему Хелли, вытекают из этой теоремы, а также приводятся примеры, иллюстрирующие ее точность.

Библиография: 16 названий.

1. Основные результаты. Цель настоящей заметки - представить новое достаточное условие на поточечно ограниченную последовательность вещественных функций $\left\{f_{j}\right\} \equiv\left\{f_{j}\right\}_{j \in \mathbb{N}}$ на отрезке $[a, b]$ вещественной прямой $\mathbb{R}$, при котором эта последовательность имеет сходящуюся всюду на $[a, b]$ подпоследовательность. Наш основной результат, теорема 1 , содержит в качестве частных случаев и классические принципы выбора Хелли для монотонных функций и функций ограниченной вариации по Жордану ([1; гл. VIII, §4]) и, как будет показано в п. 4, большинство их обобщений ([2; часть III, §2], [3]-[9] и ссылки в этих работах). Отметим, что теорема 1 остается справедливой для поточечно относительно компактной последовательности функций $\left\{f_{j}\right\}$, действующих из непустого подмножества $\mathbb{R}$ в метрическое пространство. Однако для того, чтобы представить идеи в наиболее простой форме и иметь возможность сравнения с другими принципами выбора, в этой работе рассматриваются только вещественные функции на отрезке $[a, b]$.

Для числа $\varepsilon>0$ и функции $f:[a, b] \rightarrow \mathbb{R}$ определим величину

$$
N(\varepsilon, f,[a, b]) \in\{0\} \cup \mathbb{N} \cup\{\infty\}
$$

(C) Ю.В. Третьяченко, В. В. Чистяков, 2008 
как супремум множества тех номеров $n \in \mathbb{N}$, для которых в $[a, b]$ существует набор неналегающих отрезков $\left[a_{i}, b_{i}\right], i=1, \ldots, n$, таких, что $\left|f\left(a_{i}\right)-f\left(b_{i}\right)\right|>\varepsilon$ для всех $i=1, \ldots, n$ (с соглашением о том, что $\sup \varnothing=0)$. Одним из известных свойств этой величины является следующее (теорема 2.1 в $[2$; часть III]): функция $f:[a, b] \rightarrow \mathbb{R}$ имеет односторонние конечные левый и правый пределы во всех точках отрезка $[a, b]$ тогда и только тогда, когда $N(\varepsilon, f,[a, b])<\infty$ для всех $\varepsilon>0$; в этом случае функция $f$ ограничена.

Основной результат работы - другое применение определенной выше величины $N(\varepsilon, f,[a, b])$ - это следующий поточечный принцип выбора.

Теорема 1. Пусть $\left\{f_{j}\right\}$ - поточечно ограниченная последовательность вещественных функиий на отрезке $[a, b]$ такая, что

$$
\limsup _{j \rightarrow \infty} N\left(\varepsilon, f_{j},[a, b]\right)<\infty \quad \text { для любого } \varepsilon>0 .
$$

Тогда $\left\{f_{j}\right\}$ содержит подпоследовательность, которая всюду на $[a, b]$ сходится $\kappa$ некоторой функиии $f:[a, b] \rightarrow \mathbb{R}$ такой, что $N(\varepsilon, f,[a, b])$ не превосходит верхнего предела в (1) при любом $\varepsilon>0$.

Интересно отметить, что как и ранее найденное в [7; теорема 2] и [10; лемма 4] условие (9), приводимое в п. 4.4, предположение (1) теоремы 1, является необходимым для равномерно сходящейся последовательности $\left\{f_{j}\right\}$ и "почти" необходимым для (почти) всюду сходящейся последовательности $\left\{f_{j}\right\}$ измеримых функций - это установлено в следующей ниже теореме 2. Однако предположения в большинстве известных принципов выбора (ср. с пп. 4.1-4.3 и 4.5) не являются необходимыми.

Заметим, что понятие величины $N(\varepsilon, f, E)$ для функции $f:[a, b] \rightarrow \mathbb{R}$ можно определить на любом множестве $\varnothing \neq E \subset[a, b]$, если дополнительно считать, что концы $a_{i}$ и $b_{i}$ неналегающих отрезков $\left[a_{i}, b_{i}\right], i=1, \ldots, n$, упомянутых ранее, лежат B $E$.

Теорема 2. (а) Если последовательность $\left\{f_{j}\right\}$ вещественных функций сходится равномерно на $[a, b]$ к некоторой функции $f:[a, b] \rightarrow \mathbb{R}$ такой, что $N(\varepsilon, f,[a, b])<\infty$ для всех $\varepsilon>0$, то выполнено условие (1), а точнее,

$$
\limsup _{j \rightarrow \infty} N\left(\varepsilon, f_{j},[a, b]\right) \leqslant \lim _{\delta \rightarrow \varepsilon-0} N(\delta, f,[a, b]) \quad \text { для всех } \quad \varepsilon>0 .
$$

(b) Если последовательность вещественных измеримых функций $\left\{f_{j}\right\}$ сходится на $[a, b]$ всюду (или почти всюду) к некоторой функиии $f:[a, b] \rightarrow \mathbb{R}$, удовлетворяющей для всех $\varepsilon>0$ условию $N(\varepsilon, f,[a, b])<\infty$, то для каждого $\eta>0$ в $[a, b]$ найдется измеримое по Лебегу множество $E_{\eta}$, мера которого не превосходит $\eta$, maкое, что

$$
\limsup _{j \rightarrow \infty} N\left(\varepsilon, f_{j},[a, b] \backslash E_{\eta}\right)<\infty \quad \text { для всех } \varepsilon>0 .
$$

Эти теоремы будут доказаны в следующем пункте. В п. 3 точность условий теорем 1 и 2 иллюстрируется примерами. В последнем п. 4 приводится сравнение теоремы 1 с наиболее известными к настоящему времени поточечными принципами выбора, обобщающими теорему Хелли. 


\section{2. Доказательство основных теорем.}

ДокАЗАТЕЛЬСтво тЕоремы 1. 1. Покажем, что существует подпоследовательность в $\left\{f_{j}\right\}$, снова обозначаемая через $\left\{f_{j}\right\}$, и для любого $k \in \mathbb{N}$ существует неубывающая ограниченная функция $n_{k}:[a, b] \rightarrow \mathbb{N}$ такие, что

$$
\lim _{j \rightarrow \infty} N\left(1 / k, f_{j},[a, t]\right)=n_{k}(t) \quad \text { для всех } k \in \mathbb{N} \text { и } t \in[a, b] .
$$

Прежде всего заметим, что в силу условия (1) для любого $\varepsilon>0$ найдутся такие номера $M(\varepsilon), j_{0}(\varepsilon) \in \mathbb{N}$, что $N\left(\varepsilon, f_{j},[a, b]\right) \leqslant M(\varepsilon)$ для всех $j \geqslant j_{0}(\varepsilon)$. Последовательность

$$
\left\{t \mapsto N\left(1, f_{j},[a, t]\right)\right\}_{j \geqslant j_{0}(1)},
$$

состоящая из неубывающих функций, равномерно ограничена на $[a, b]$ постоянной $M(1)$. По принципу выбора Хелли для монотонных функций в последовательности $\left\{f_{j}\right\}_{j \geqslant j_{0}(1)}$, и, тем самым, в исходной последовательности $\left\{f_{j}\right\}$, найдется подпоследовательность $\left\{f_{J_{1}(j)}\right\}_{j=1}^{\infty}$ (здесь последовательность $J_{1}: \mathbb{N} \rightarrow \mathbb{N}$, также, как и встречающиеся ниже подобные ей последовательности $J_{k}$ при $k \geqslant 2$, является строго возрастающей) такая, что $N\left(1, f_{J_{1}(j)},[a, t]\right)$ сходится при $j \rightarrow \infty$ к $n_{1}(t)$ для всех $t \in[a, b]$, где $n_{1}:[a, b] \rightarrow \mathbb{N}$ - некоторая неубывающая ограниченная функция. Выберем наименьший номер $j_{1} \in \mathbb{N}$ такой, что $J_{1}\left(j_{1}\right) \geqslant j_{0}(1 / 2)$. Тогда последовательность неубывающих функций

$$
\left\{t \mapsto N\left(1 / 2, f_{J_{1}(j)},[a, t]\right)\right\}_{j \geqslant j_{1}}
$$

равномерно ограничена на $[a, b]$ постоянной $M(1 / 2)$. Снова применяя принцип выбора Хелли, в $\left\{f_{J_{1}(j)}\right\}_{j \geqslant j_{1}}$ найдем подпоследовательность $\left\{f_{J_{2}(j)}\right\}_{j=1}^{\infty}$ такую, что $N\left(1 / 2, f_{J_{2}(j)},[a, t]\right)$ сходится при $j \rightarrow \infty$ к $n_{2}(t)$ для всех $t \in[a, b]$, где $n_{2}:[a, b] \rightarrow \mathbb{N}-$ также неубывающая ограниченная функция. Выберем минимальный номер $j_{2} \in \mathbb{N}$ такой, что $J_{2}\left(j_{2}\right) \geqslant j_{0}(1 / 3)$. Теперь по индукции предположим, что для номера $k \geqslant 3$ подпоследовательность $\left\{f_{J_{k-1}(j)}\right\}_{j=1}^{\infty}$ в исходной последовательности $\left\{f_{j}\right\}$ и наименьший номер $j_{k-1} \in \mathbb{N}$ такой, что $J_{k-1}\left(j_{k-1}\right) \geqslant j_{0}(1 / k)$, уже выбраны. Применим теорему Хелли к последовательности неубывающих функций

$$
\left\{t \mapsto N\left(1 / k, f_{J_{k-1}(j)},[a, t]\right)\right\}_{j \geqslant j_{k-1}},
$$

которая равномерно ограничена на $[a, b]$ постоянной $M(1 / k)$ : существуют подпоследовательность $\left\{f_{J_{k}(j)}\right\}_{j=1}^{\infty}$ последовательности $\left\{f_{J_{k-1}(j)}\right\}_{j \geqslant j_{k-1}}$ и неубывающая ограниченная функция $n_{k}:[a, b] \rightarrow \mathbb{N}$ такие, что $N\left(1 / k, f_{J_{k}(j)},[a, t]\right)$ сходится при $j \rightarrow \infty$ к $n_{k}(t)$ для всех $t \in[a, b]$. Замечая, что при любом $k \in \mathbb{N}$ последовательность $\left\{f_{J_{j}(j)}\right\}_{j \geqslant k}$ является подпоследовательностью в $\left\{f_{J_{k}(j)}\right\}_{j=1}^{\infty}$, в силу приведенных рассуждений найдем, что диагональная последовательность $\left\{f_{J_{j}(j)}\right\}_{j=1}^{\infty}$, снова обозначаемая через $\left\{f_{j}\right\}$, удовлетворяет условию (2).

2. При любом $k \in \mathbb{N}$ множество $S_{k} \subset[a, b]$ точек разрыва монотонной функции $n_{k}$ не более чем счетно. Положим $S=([a, b] \cap \mathbb{Q}) \cup \bigcup_{k=1}^{\infty} S_{k}$, где $\mathbb{Q}$ - множество всех рациональных чисел. Тогда $S$ - счетное всюду плотное подмножество $[a, b]$ такое, что

функция $n_{k}$ непрерывна на $[a, b] \backslash S$ при любом $k \in \mathbb{N}$. 
Поскольку последовательность $\left\{f_{j}\right\}$ поточечно ограничена и $S$ счетно, без ограничения общности предполагаем (при необходимости переходя к подпоследовательности в $\left\{f_{j}\right\}$ при помощи стандартного диагонального процесса), что при каждом $s \in S$ последовательность $\left\{f_{j}(s)\right\}$ сходится в $\mathbb{R}$ при $j \rightarrow \infty$ к некоторой точке, обозначаемой через $f(s)$.

Покажем теперь, что последовательность $\left\{f_{j}(t)\right\}$ фундаментальна в любой точке $t \in[a, b] \backslash S$. Пусть $\varepsilon>0$ произвольно. Выберем и зафиксируем номер $k=k(\varepsilon) \in \mathbb{N}$ такой, что $1 / k \leqslant \varepsilon / 3$. Так как в силу (3) $t$ есть точка непрерывности функции $n_{k}$ и $S$ всюду плотно в $[a, b]$, найдется точка $s=s(k, t) \in S$, таким образом зависящая лишь от $\varepsilon$, такая, что $n_{k}(t)=n_{k}(s)$. Пусть для определенности $s<t$ (случай, когда $t<s$, аналогичен). Используя (2), выберем номера $J_{1}=J_{1}(k, t), J_{2}=J_{2}(k, s) \in \mathbb{N}$, также зависящие лишь от $\varepsilon$, такие, что $N\left(1 / k, f_{j},[a, t]\right)=n_{k}(t)$ для всех $j \geqslant J_{1}$ и $N\left(1 / k, f_{j},[a, s]\right)=n_{k}(s)$ для всех $j \geqslant J_{2}$. Тогда при $j \geqslant \max \left\{J_{1}, J_{2}\right\}$ получим, что

$$
N\left(\frac{1}{k}, f_{j},[s, t]\right) \leqslant N\left(\frac{1}{k}, f_{j},[a, t]\right)-N\left(\frac{1}{k}, f_{j},[a, s]\right)=n_{k}(t)-n_{k}(s)=0,
$$

откуда $N\left(1 / k, f_{j},[s, t]\right)=0$, что благодаря определению величины слева в последнем равенстве означает, в частности, что $\left|f_{j}(s)-f_{j}(t)\right| \leqslant 1 / k \leqslant \varepsilon / 3$. Поскольку последовательность $\left\{f_{j}(s)\right\}$ сходится, она фундаментальна, поэтому существует номер $J_{3}=J_{3}(\varepsilon, s) \in \mathbb{N}$ такой, что $\left|f_{j}(s)-f_{l}(s)\right| \leqslant \varepsilon / 3$ для всех $j, l \geqslant J_{3}$. Следовательно, номер $J=\max \left\{J_{1}, J_{2}, J_{3}\right\}$ зависит только от $\varepsilon$, и для всех $j, l \geqslant J$ находим, что

$$
\left|f_{j}(t)-f_{l}(t)\right| \leqslant\left|f_{j}(t)-f_{j}(s)\right|+\left|f_{j}(s)-f_{l}(s)\right|+\left|f_{l}(s)-f_{l}(t)\right| \leqslant \varepsilon .
$$

Из фундаментальности последовательности $\left\{f_{j}(t)\right\}$ вытекает ее сходимость. Обозначим предел этой последовательности через $f(t)$. Тем самым определена функция $f:[a, b]=S \cup([a, b] \backslash S) \rightarrow \mathbb{R}$, к которой всюду на $[a, b]$ сходится последовательность $\left\{f_{j}\right\}$, являющаяся подпоследовательностью исходной последовательности $\left\{f_{j}\right\}$. Осталось заметить, что

$$
N(\varepsilon, f,[a, b]) \leqslant \liminf _{j \rightarrow \infty} N\left(\varepsilon, f_{j},[a, b]\right) \leqslant \limsup _{j \rightarrow \infty} N\left(\varepsilon, f_{j},[a, b]\right)
$$

для всех $\varepsilon>0$, и установить здесь неравенство слева.

Для этого без ограничения общности считаем, что $N(\varepsilon, f,[a, b])>0$. Достаточно показать, что если номер $n \in \mathbb{N}$ такой, что в $[a, b]$ найдутся неналегающие отрезки $\left[a_{i}, b_{i}\right], i=1, \ldots, n$, для которых $\left|f\left(a_{i}\right)-f\left(b_{i}\right)\right|>\varepsilon$ для всех $i=1, \ldots, n$, то

$$
n \leqslant \liminf _{j \rightarrow \infty} N\left(\varepsilon, f_{j},[a, b]\right) .
$$

Для любого числа $\varepsilon^{\prime}=\varepsilon^{\prime}(n)$ со свойством $\min _{1 \leqslant i \leqslant n}\left|f\left(a_{i}\right)-f\left(b_{i}\right)\right|>\varepsilon^{\prime}>\varepsilon$ в силу сходимости всюду на $[a, b] f_{j}$ к $f$ существует номер $J=J(n) \in \mathbb{N}$ такой, что значения $\left|f\left(a_{i}\right)-f_{j}\left(a_{i}\right)\right|$ и $\left|f_{j}\left(b_{i}\right)-f\left(b_{i}\right)\right|$ не превосходят $\left(\varepsilon^{\prime}-\varepsilon\right) / 2$ для всех $i=1, \ldots, n$ и $j \geqslant J$. Отсюда для этих же $i$ и $j$ найдем, что

$$
\begin{aligned}
\varepsilon^{\prime} & <\left|f\left(a_{i}\right)-f\left(b_{i}\right)\right| \leqslant\left|f\left(a_{i}\right)-f_{j}\left(a_{i}\right)\right|+\left|f_{j}\left(a_{i}\right)-f_{j}\left(b_{i}\right)\right|+\left|f_{j}\left(b_{i}\right)-f\left(b_{i}\right)\right| \\
& \leqslant \frac{\varepsilon^{\prime}-\varepsilon}{2}+\left|f_{j}\left(a_{i}\right)-f_{j}\left(b_{i}\right)\right|+\frac{\varepsilon^{\prime}-\varepsilon}{2}=\left|f_{j}\left(a_{i}\right)-f_{j}\left(b_{i}\right)\right|+\varepsilon^{\prime}-\varepsilon,
\end{aligned}
$$


а потому, $\left|f_{j}\left(a_{i}\right)-f_{j}\left(b_{i}\right)\right|>\varepsilon, i=1, \ldots, n$. По определению $N\left(\varepsilon, f_{j},[a, b]\right)$ тогда имеем $n \leqslant N\left(\varepsilon, f_{j},[a, b]\right)$ для всех $j \geqslant J$ и, следовательно,

$$
n \leqslant \inf _{j \geqslant J} N\left(\varepsilon, f_{j},[a, b]\right) \leqslant \liminf _{j \rightarrow \infty} N\left(\varepsilon, f_{j},[a, b]\right),
$$

что завершает доказательство неравенства и вместе с ним теоремы 1.

ДОКАЗАТЕЛЬСТво тЕОРЕмЫ 2. (а) По условию функция $f$ ограничена (см. п. 1 ), а из равномерной сходимости $\left\{f_{j}\right\}$ к $f$ вытекает, что

$$
\sup _{s, t \in[a, b]}\left|\left(f_{j}-f\right)(s)-\left(f_{j}-f\right)(t)\right| \leqslant 2 \sup _{t \in[a, b]}\left|f_{j}(t)-f(t)\right| \rightarrow 0 \quad \text { при } \quad j \rightarrow \infty .
$$

Кроме того, для любых $\varepsilon>0$ и $j \in \mathbb{N}$ справедливо неравенство

$$
N\left(\varepsilon, f_{j},[a, b]\right) \leqslant N\left(\varepsilon-\delta, f_{j}-f,[a, b]\right)+N(\delta, f,[a, b]) \quad \text { для всех } \quad 0<\delta<\varepsilon .
$$

Действительно, без потери общности считая, что левая часть неравенства в (5) больше нуля, предположим, что номер $n \in \mathbb{N}$ такой, что существует набор $\left[a_{i}, b_{i}\right]$, $i=1, \ldots, n$, неналегающих отрезков в $[a, b]$, для которого $\left|f_{j}\left(a_{i}\right)-f_{j}\left(b_{i}\right)\right|>\varepsilon$ для всех $i=1, \ldots, n$. Поскольку для таких $i$ имеем

$$
\varepsilon<\left|f_{j}\left(a_{i}\right)-f_{j}\left(b_{i}\right)\right| \leqslant\left|\left(f_{j}-f\right)\left(a_{i}\right)-\left(f_{j}-f\right)\left(b_{i}\right)\right|+\left|f\left(a_{i}\right)-f\left(b_{i}\right)\right|,
$$

то $\left|\left(f_{j}-f\right)\left(a_{i}\right)-\left(f_{j}-f\right)\left(b_{i}\right)\right|>\varepsilon-\delta$ или $\left|f\left(a_{i}\right)-f\left(b_{i}\right)\right|>\delta$; в первом случае отнесем индекс $i$ к множеству $I_{1} \subset\{1, \ldots, n\}$, а во втором случае - к множеству $I_{2} \subset$ $\{1, \ldots, n\}$, и обозначим через $\left|I_{1}\right|$ и $\left|I_{2}\right|$ количество (возможно нулевое) элементов соответственно в $I_{1}$ и $I_{2}$. Ясно, что $\left|I_{1}\right|+\left|I_{2}\right| \geqslant n$. С другой стороны, из определения величины $N(\cdots)$ в п. 1 следует, что

$$
\left|I_{1}\right| \leqslant N\left(\varepsilon-\delta, f_{j}-f,[a, b]\right) \quad \text { и } \quad\left|I_{2}\right| \leqslant N(\delta, f,[a, b]) .
$$

Таким образом, $n \leqslant N\left(\varepsilon-\delta, f_{j}-f,[a, b]\right)+N(\delta, f,[a, b])$, откуда получаем (5) в силу произвольности упомянутых номеров $n$.

Пусть теперь $\varepsilon>0$ и $0<\delta<\varepsilon$ произвольны. Найдется такой номер $J \in \mathbb{N}$, зависящий от $\varepsilon$ и $\delta$, что $\left|\left(f_{j}-f\right)(s)-\left(f_{j}-f\right)(t)\right| \leqslant \varepsilon-\delta$ для всех $s, t \in[a, b]$ и $j \geqslant J$. Отсюда по определению $N(\cdot)$ первое слагаемое справа в неравенстве (5) обращается в нуль и, значит, $N\left(\varepsilon, f_{j},[a, b]\right) \leqslant N(\delta, f,[a, b])$ для всех $j \geqslant J$. Следовательно,

$$
\limsup _{j \rightarrow \infty} N\left(\varepsilon, f_{j},[a, b]\right) \leqslant \sup _{j \geqslant J} N\left(\varepsilon, f_{j},[a, b]\right) \leqslant N(\delta, f,[a, b]) .
$$

Осталось принять во внимание невозрастание функции $\delta \mapsto N(\delta, f,[a, b])$ и перейти к пределу в последнем неравенстве при $\delta \rightarrow \varepsilon-0$.

(b) По теореме Егорова [1; гл. IV, §3] из сходимости (почти) всюду $\left\{f_{j}\right\}$ к $f$ вытекает, что для любого $\eta>0$ в $[a, b]$ существует такое измеримое множество $E_{\eta}$ меры $\leqslant \eta$, что $\left\{f_{j}\right\}$ сходится к $f$ на $[a, b] \backslash E_{\eta}$ равномерно. Заменяя в рассуждениях (а) отрезок $[a, b]$ на $[a, b] \backslash E_{\eta}$, придем к оценке

$$
\limsup _{j \rightarrow \infty} N\left(\varepsilon, f_{j},[a, b] \backslash E_{\eta}\right) \leqslant \lim _{\delta \rightarrow \varepsilon-0} N\left(\delta, f,[a, b] \backslash E_{\eta}\right) \leqslant \lim _{\delta \rightarrow \varepsilon-0} N(\delta, f,[a, b])<\infty
$$

для любого $\varepsilon>0$, что и требовалось установить. 
3. Примеры. В этом разделе собраны некоторые примеры последовательностей функций $\left\{f_{j}\right\}$ на отрезке $[0,1]$ (кроме пп. 3.2 и 3.4 , где $[a, b]=[0,2 \pi]$ ), показывающие точность предположений и заключений теорем 1 и 2.

Поскольку колебание $\operatorname{osc}(f,[a, b])=\sup _{s, t \in[a, b]}|f(s)-f(t)|$ ограниченной функции $f:[a, b] \rightarrow \mathbb{R}$ конечно, имеем: если оsc $(f,[a, b])=0$, то $f$ постоянна, откуда $N(\varepsilon, f,[a, b])=0$ для всех $\varepsilon>0$, а если $\varepsilon \geqslant \operatorname{osc}(f,[a, b])>0$, то $N(\varepsilon, f,[a, b])=0$ по определению. Поэтому ниже оценки для $N(\varepsilon, f,[a, b])$ будут выписываться только для случая, когда $0<\varepsilon<\operatorname{osc}(f,[a, b])$. Например, если $V_{a}^{b}(f)$ обозначает обычную вариацию по Жордану функции $f$ на отрезке $[a, b]$ (см. также п. 4.1 при $\varphi(t, u)=u$, то

$$
\operatorname{osc}(f,[a, b]) \leqslant V_{a}^{b}(f) \quad \text { и } \quad N(\varepsilon, f,[a, b]) \leqslant \frac{1}{\varepsilon} V_{a}^{b}(f) \quad \text { при } \quad 0<\varepsilon<\operatorname{osc}(f,[a, b]) .
$$

3.1. Условие поточечной ограниченности $\left\{f_{j}\right\}$ в теореме 1 существенно: последовательность $f_{j}(t)=0$ при $0 \leqslant t<1$ и $f_{j}(1)=j$ не имеет всюду сходящейся подпоследовательности, однако, $N\left(\varepsilon, f_{j},[0,1]\right)=1$ при $0<\varepsilon<j$, а потому

$$
\limsup _{j \rightarrow \infty} N\left(\varepsilon, f_{j},[0,1]\right)=1 \quad \text { для всех } \varepsilon>0 .
$$

В то же время условие поточечной ограниченности не является необходимым: последовательность $f_{j}(t)=0$ при $0 \leqslant t<1$ и $f_{j}(1)=j^{(-1)^{j}}$ содержит всюду сходящуюся подпоследовательность, соответствующую нечетным номерам $j$, и является неограниченной в точке $t=1$, причем, как и выше,

$$
\limsup _{j \rightarrow \infty} N\left(\varepsilon, f_{j},[0,1]\right)=1 \quad \text { для всех } \varepsilon>0 .
$$

3.2. Без предположения (1) теорема 1 не имеет места. Действительно, известно, что последовательность $f_{j}(t)=\sin (j t), 0 \leqslant t \leqslant 2 \pi$, не имеет ни одной подпоследовательности, которая сходится всюду на $[0,2 \pi]$. С другой стороны, покажем, что

$$
4 j \leqslant N\left(\varepsilon, f_{j},[0,2 \pi]\right) \leqslant \frac{4 j}{\varepsilon} \quad \text { для всех } 0<\varepsilon<1 .
$$

Поскольку $\operatorname{osc}\left(f_{j},[0,2 \pi]\right)=2$ и $V_{0}^{2 \pi}\left(f_{j}\right)=4 j$, в силу (6) при $0<\varepsilon<2$ получим неравенство справа. Пусть теперь $0<\varepsilon<1$. Для $j \in \mathbb{N}$ положим $t_{i}^{j}=\pi i /(2 j)$, $i=0,1, \ldots, 4 j$, и $\left[a_{i}, b_{i}\right]=\left[t_{i-1}^{j}, t_{i}^{j}\right], i=1, \ldots, 4 j$. Тогда для всех $i=1, \ldots, 4 j$ найдем, что

$$
\left|f_{j}\left(a_{i}\right)-f_{j}\left(b_{i}\right)\right|=\left|\sin \left(\frac{\pi i}{2}\right)-\sin \left(\frac{\pi(i-1)}{2}\right)\right|=1>\varepsilon,
$$

откуда по определению величины $N(\cdot)$ вытекает неравенство слева в (7), из которого следует, что $\lim \sup _{j \rightarrow \infty} N\left(\varepsilon, f_{j},[0,2 \pi]\right)=\infty$ для всех $0<\varepsilon<1$.

3.3. Условие (1) не является необходимым, так что теорема 2, (а) нарушается для всюду сходящейся последовательности $\left\{f_{j}\right\}$. Пусть $\mathscr{D}$ есть функция Дирихле: $\mathscr{D}(t)=1$ при $t \in[0,1] \cap \mathbb{Q}$ и $\mathscr{D}(t)=0$ при $t \in[0,1] \backslash \mathbb{Q}$. Тогда $N(\varepsilon, \mathscr{D},[0,1])=\infty$ при $0<\varepsilon<1=\operatorname{osc}(\mathscr{D},[0,1])$. На $[0,1]$ рассмотрим последовательность

$$
f_{j}(t)=\lim _{m \rightarrow \infty}(\cos (j ! \pi t))^{2 m}
$$


она принимает значение 1 , если $j ! t$ - целое число, и значение 0 в противном случае, а потому всюду на $[0,1]$ сходится к функции Дирихле $\mathscr{D}$. Поскольку osc $\left(f_{j},[0,1]\right)=1$ и $V_{0}^{1}\left(f_{j}\right)=2 \cdot j$ !, то $N\left(\varepsilon, f_{j},[0,1]\right)=2 \cdot j$ ! при $0<\varepsilon<1$, так что

$$
\limsup _{j \rightarrow \infty} N\left(\varepsilon, f_{j},[0,1]\right)=\infty \quad \text { для всех } \quad 0<\varepsilon<1 .
$$

Отметим, что здесь также показано, что для всюду сходящейся последовательности $\left\{f_{j}\right\}$ из условия $N\left(\varepsilon, f_{j},[0,1]\right)<\infty$ для всех $\varepsilon>0$ и $j \in \mathbb{N}$ не следует, что

$$
\limsup _{j \rightarrow \infty} N\left(\varepsilon, f_{j},[0,1]\right)<\infty \quad \text { для всех } \varepsilon>0 .
$$

Кроме того, возможен случай, когда все функции $f_{j}$ последовательности $\left\{f_{j}\right\}$ не удовлетворяют условию $N\left(\varepsilon, f_{j},[a, b]\right)<\infty$ для всех $\varepsilon>0$, тогда как предельная функция $f$ из теоремы 1 всегда этому условию удовлетворяет: последовательность $f_{j}(t)=\mathscr{D}(t) / j$ равномерно на $[0,1]$ сходится к нулевой функции, $N\left(\varepsilon, f_{j},[0,1]\right)=\infty$ при $0<\varepsilon<1 / j=\operatorname{osc}\left(f_{j},[0,1]\right)$ и $N\left(\varepsilon, f_{j},[0,1]\right)=0$ для всех $\varepsilon>0$ и $j \geqslant 1 / \varepsilon$.

3.4. Другим примером всюду сходящейся (к нулю) последовательности $\left\{f_{j}\right\}$, для которой нарушается условие $(1)$, служит пример из [10; пример 4]: положив $f_{j}(t)=$ $\sin \left(j^{2} t\right)$ при $0 \leqslant t \leqslant 2 \pi / j$ и $f_{j}(t)=0$ при $2 \pi / j \leqslant t \leqslant 2 \pi$, найдем, что выполняются неравенства (7).

3.5. Левый предел при $\delta \rightarrow \varepsilon-0$ в неравенстве теоремы 2 , (а) нельзя заменить выражением $N(\varepsilon, f,[a, b])$ : последовательность $f_{j}(t)=0$ при $0 \leqslant t<1$ и $f_{j}(1)=$ $1+(1 / j)$ сходится равномерно на $[0,1]$ к функции $f(t)=0$ при $0 \leqslant t<1$ и $f(1)=1$, так что при $\varepsilon=1$ имеем $N\left(\varepsilon, f_{j},[0,1]\right)=1$ для всех $j \in \mathbb{N}$ и $N(\delta, f,[0,1])=1$ для всех $0<\delta<\varepsilon$, тогда как $N(\varepsilon, f,[0,1])=0$.

3.6. Для всюду сходящейся последовательности $\left\{f_{j}\right\}$ условие (1) может быть выполнено, а неравенство в теореме $2,\left(\right.$ a) - нет: последовательность $f_{j}(t)=1$ при $t=1 /(j+1)$ и $f_{j}(t)=0$ при $t \neq 1 /(j+1)$ сходится всюду на $[0,1]$ к функции $f(0)=1$ и $f(t)=0$ при $0<t \leqslant 1$, причем если $0<\varepsilon<1$, то $N\left(\varepsilon, f_{j},[0,1]\right)=2$ для всех $j \in \mathbb{N}$ и $N(\varepsilon, f,[0,1])=1$.

3.7. Здесь приводится пример последовательности $\left\{f_{j}\right\}$ на $[0,1]$, которая не ограничена равномерно и для которой применима теорема 1 . Положим $f_{j}(t)=j$ при $t=1 /(j+1)$ и $f_{j}(t)=0$ при $t \neq 1 /(j+1)$. Тогда $\left\{f_{j}\right\}$ всюду сходится к $f \equiv 0$, поэтому $\left\{f_{j}\right\}$ поточечно ограничена; более того, $\left\{f_{j}(t)\right\}=\{0\}$ при $t \notin\{1 /(k+1)\}_{k \in \mathbb{N}}$ и $\left\{f_{j}(t)\right\}=\{0, k\}$ при $t=1 /(k+1), k \in \mathbb{N}$. Далее, $\operatorname{osc}\left(f_{j},[0,1]\right)=j$ (стремится к бесконечности при $j \rightarrow \infty)$ и в силу $(6) N\left(\varepsilon, f_{j},[0,1]\right)=2$ при $0<\varepsilon<j$, откуда

$$
\limsup _{j \rightarrow \infty} N\left(\varepsilon, f_{j},[0,1]\right)=2 \quad \text { для всех } \varepsilon>0 .
$$

3.8. Существуют всюду сходящиеся к нулю последовательности $\left\{f_{j}\right\}$ (а потому, поточечно ограниченные), для которых нарушается условие (1) и

$$
\limsup _{j \rightarrow \infty} \operatorname{osc}\left(f_{j},[0,1]\right)=\infty .
$$


Положим $f_{j}(t)=j t^{j}$ при $0 \leqslant t<1$ и $f_{j}(1)=0$. Тогда $\operatorname{osc}\left(f_{j},[0,1]\right)=j, V_{0}^{1}\left(f_{j}\right)=2 j$ и, потому, в силу $(6) N\left(\varepsilon, f_{j},[0,1]\right) \leqslant 2 j / \varepsilon$ при $0<\varepsilon<j$. Покажем, что

$$
N\left(\varepsilon, f_{j},[0,1]\right) \geqslant[j /(2 \varepsilon)] \quad \text { при } \quad j>2 \varepsilon
$$

(здесь квадратные скобки обозначают целую часть числа), откуда будет следовать, что

$$
\limsup _{j \rightarrow \infty} N\left(\varepsilon, f_{j},[0,1]\right)=\infty \quad \text { для всех } \varepsilon>0 .
$$

Действительно, для $j>2 \varepsilon$ положим $t_{i}=(2 i \varepsilon / j)^{1 / j}, i=0,1, \ldots,[j /(2 \varepsilon)]$, и $\left[a_{i}, b_{i}\right]=$ $\left[t_{i-1}, t_{i}\right], i=1, \ldots,[j /(2 \varepsilon)]$. Тогда $0=t_{0}<t_{1}<\cdots<t_{[j /(2 \varepsilon)]} \leqslant 1$ и

$$
\left|f_{j}\left(a_{i}\right)-f_{j}\left(b_{i}\right)\right|=j\left(t_{i}\right)^{j}-j\left(t_{i-1}\right)^{j}=j \frac{2 i \varepsilon}{j}-j \frac{2(i-1) \varepsilon}{j}=2 \varepsilon>\varepsilon
$$

для всех $i=1, \ldots,[j /(2 \varepsilon)]$, откуда следует искомое неравенство.

3.9. В определении величины $N(\varepsilon, f,[a, b])$ в п. 1 требуется, чтобы неналегающие отрезки $\left[a_{i}, b_{i}\right]$ обладали свойством: $\left|f\left(a_{i}\right)-f\left(b_{i}\right)\right|>\varepsilon$ для всех $i=1, \ldots, n$. Если здесь заменить неравенство $>\varepsilon$ на $\geqslant \varepsilon$ и новую величину обозначить через $N_{\geqslant}(\varepsilon, f,[a, b])$, то для нее неравенство слева в (4) может нарушаться: последовательность $f_{j}(t)=1$ при $t=0, f_{j}(t)=0$ при $0<t<1$ и $f_{j}(t)=1-(1 / j)$ при $t=1$ сходится равномерно на $[0,1]$ к функции $f(0)=f(1)=1$ и $f(t)=0$ при $0<t<1$, и при $\varepsilon=1$ найдем, что $N_{\geqslant}\left(\varepsilon, f_{j},[0,1]\right)=1$ для всех $j \in \mathbb{N}$ и $N_{\geqslant}(\varepsilon, f,[0,1])=2$.

\section{4. Сравнение с известными принципами выбора.}

4.1. Пусть функция $\varphi:[a, b] \times \mathbb{R}^{+} \rightarrow \mathbb{R}^{+}$, где $\mathbb{R}^{+}=[0, \infty)$, удовлетворяет следующим двум условиям:

(i) при любом $t \in[a, b]$ функция $\varphi(t, \cdot)$ второго аргумента не убывает и непрерывна на $\mathbb{R}^{+}$и $\varphi(t, u) \rightarrow \infty$ при $u \rightarrow \infty$;

(ii) $\varphi(t, 0)=0$ при любом $t \in[a, b]$ и $\inf _{t \in[a, b]} \varphi(t, u)>0$ для любого $u>0$. Следуя [3], для функции $f:[a, b] \rightarrow \mathbb{R}$ положим

$$
V_{\varphi}(f,[a, b])=\sup \sum_{i=1}^{n} \varphi\left(t_{i},\left|f\left(a_{i}\right)-f\left(b_{i}\right)\right|\right)
$$

где супремум берется по всем $n \in \mathbb{N}$, всем наборам $\left[a_{i}, b_{i}\right], i=1, \ldots, n$, неналегающих отрезков из $[a, b]$ и всем точкам $t_{i} \in\left[a_{i}, b_{i}\right], i=1, \ldots, n$. Эта обобщенная вариация функции $f$ соответствует $\varphi$-вариации по Винеру-Янгу при $\varphi(t, u)=\varphi(u)$ и обычной вариации по Жордану $V_{a}^{b}(f)$ при $\varphi(t, u)=u$. В цитированной работе установлено следующее обобщение теоремы Хелли: если последовательность функиий $\left\{f_{j}\right\}$ на отрезке $[a, b]$ ограничена в некоторой точке из $[a, b] u$

$$
\sup _{j \in \mathbb{N}} V_{\varphi}\left(f_{j},[a, b]\right)=C<\infty
$$

то $\left\{f_{j}\right\}$ содержит подпоследовательность, которая всюду на $[a, b]$ сходится к некоторой функции $f$ такой, что $V_{\varphi}(f,[a, b]) \leqslant C$. Этот результат сразу вытекает из 
теоремы 1 (вместе с теоремой Хелли при $\varphi(t, u)=u$ и ее обобщением из [4; теорема 1.3] при $\varphi(t, u)=\varphi(u))$, если принять во внимание оценки (ср. с оценками из доказательства теоремы 9 из [12]):

$$
\begin{gathered}
\operatorname{osc}\left(f_{j},[a, b]\right) \leqslant 2 \max \left\{u \in \mathbb{R}^{+} \mid \varphi(a, u) \leqslant V_{\varphi}\left(f_{j},[a, b]\right)\right\}, \\
N\left(\varepsilon, f_{j},[a, b]\right) \leqslant \frac{V_{\varphi}\left(f_{j},[a, b]\right)}{\inf _{t \in[a, b]} \varphi(t, \varepsilon)} \quad \text { для } \quad 0<\varepsilon<\operatorname{osc}\left(f_{j},[a, b]\right),
\end{gathered}
$$

а также секвенциальную полунепрерывность снизу функционала $V_{\varphi}$ : если последовательность $\left\{f_{j}\right\}$ сходится всюду на $[a, b]$ к функции $f$, то

$$
V_{\varphi}(f,[a, b]) \leqslant \liminf _{j \rightarrow \infty} V_{\varphi}\left(f_{j},[a, b]\right) \leqslant C .
$$

4.2. Пусть $\Phi=\left\{\varphi_{i}\right\}_{i \in \mathbb{N}}-$ последовательность, состоящая из непрерывных неубывающих неограниченных функций $\varphi_{i}: \mathbb{R}^{+} \rightarrow \mathbb{R}^{+}$таких, что $\varphi_{i}(u)=0$ лишь при $u=0$, и удовлетворяющих следующим двум условиям:

(a) $\varphi_{i+1}(u) \leqslant \varphi_{i}(u)$ для всех $i \in \mathbb{N}$ и $u \in \mathbb{R}^{+}$;

(b) $\sum_{i=1}^{\infty} \varphi_{i}(u)=\infty$ для всех $u>0$.

Следуя [8], для функции $f:[a, b] \rightarrow \mathbb{R}$ положим

$$
V_{\Phi}(f,[a, b])=\sup \sum_{i=1}^{n} \varphi_{i}\left(\left|f\left(a_{i}\right)-f\left(b_{i}\right)\right|\right),
$$

где супремум берется по всем $n \in \mathbb{N}$ и всем неупорядоченным наборам $\left[a_{i}, b_{i}\right]$, $i=1, \ldots, n$, неналегающих отрезков из $[a, b]$. Такая обобщенная вариация функции $f$ соответствует вариации по Жордану $V_{a}^{b}(f)$ при $\varphi_{i}(u)=u, \varphi$-вариации по Винеру-Янгу при $\varphi_{i}(u)=\varphi(u)$ и $\Lambda$-вариации по Уотерману [9] при $\varphi_{i}(u)=u / \lambda_{i}$ для всех $i \in \mathbb{N}$ и $u \in \mathbb{R}^{+}$, где $\Lambda=\left\{\lambda_{i}\right\}_{i \in \mathbb{N}}$ - неубывающая последовательность положительных чисел, для которой $\sum_{i=1}^{\infty} 1 / \lambda_{i}=\infty$. Обобщение теоремы Хелли из [8; теорема 2.8] (и его частный случай из [9; теорема 5]) формулируется так же, как соответствующее утверждение в п. 4.1 с заменой $V_{\varphi}$ на $V_{\Phi}$. Этот результат вытекает из теоремы 1 , благодаря следующим оценкам (ср. с оценками из доказательства теоремы 10 в [12]):

$$
\begin{gathered}
\operatorname{osc}\left(f_{j},[a, b]\right) \leqslant \max \left\{u \in \mathbb{R}^{+} \mid \varphi_{1}(u) \leqslant V_{\Phi}\left(f_{j},[a, b]\right)\right\}, \\
N\left(\varepsilon, f_{j},[a, b]\right) \leqslant \max \left\{n \in \mathbb{N} \mid \sum_{i=1}^{n} \varphi_{i}(\varepsilon) \leqslant V_{\Phi}\left(f_{j},[a, b]\right)\right\} \quad \text { при } \quad 0<\varepsilon<\operatorname{osc}\left(f_{j},[a, b]\right),
\end{gathered}
$$

а также свойству секвенциальной полунепрерывности снизу функционала $V_{\Phi}$.

4.3. Из теоремы 1 вытекает и установленный другим способом принцип выбора типа Хелли из работы [2; часть III, §2, предложение 2.8], в котором для справедливости заключения теоремы 1 предполагается значительно больше, а именно, что последовательность $\left\{f_{j}\right\}$ равномерно ограничена,

$$
\sup _{j \in \mathbb{N}} \operatorname{osc}\left(f_{j},[a, b]\right)<\infty \quad \text { и } \quad \sup _{j \in \mathbb{N}} N\left(\varepsilon, f_{j},[a, b]\right)<\infty \quad \text { для всех } \quad \varepsilon>0 .
$$


4.4. Напомним, что модулем изменения по Чантурия [13] функции $f:[a, b] \rightarrow \mathbb{R}$ называется последовательность $\{\nu(n, f,[a, b])\}_{n \in \mathbb{N}}$, определяемая правилом

$$
\nu(n, f,[a, b])=\sup \sum_{i=1}^{n}\left|f\left(a_{i}\right)-f\left(b_{i}\right)\right|,
$$

где для фиксированного $n \in \mathbb{N}$ супремум берется по всем наборам $\left[a_{i}, b_{i}\right], i=1, \ldots, n$, неналегающих отрезков из $[a, b]$. Как отмечено в [13; теорема 5] (см. также [7; теорема 3]), функция $f:[a, b] \rightarrow \mathbb{R}$ имеет односторонние конечные левый и правый пределы во всех точках $[a, b]$ тогда и только тогда, когда $\nu(n, f,[a, b])=o(n)$ (т.е. $\nu(n, f,[a, b]) / n \rightarrow 0$ при $n \rightarrow \infty)$. Отметим [2; часть III, §2, теорема 2.2], что для последовательности функций $\left\{f_{j}\right\}$ условия (8) эквивалентны одному условию:

$$
\sup _{j \in \mathbb{N}} \nu\left(n, f_{j},[a, b]\right)=o(n) .
$$

В работе [7; теорема 1] (и ее обобщениях [10]-[12]) установлен следующий принцип выбора, обобщающий теорему Хелли: если последовательность функиий $\left\{f_{j}\right\}$ на $[a, b]$ ограничена в некоторой точке из $[a, b]$ u

$$
\limsup _{j \rightarrow \infty} \nu\left(n, f_{j},[a, b]\right)=o(n),
$$

то $\left\{f_{j}\right\}$ содержит подпоследовательность, которая всюду на $[a, b]$ сходится $к$ некоторой функиии $f$ такой, что $\nu(n, f,[a, b])=o(n)$. Этот результат вытекает из теоремы 1 , если учесть, что условие (9) эквивалентно сразу паре условий - условию (1) и $\limsup _{j \rightarrow \infty} \operatorname{osc}\left(f_{j},[a, b]\right)<\infty$. Заметим, что во всех приведенных выше обобщениях теоремы Хелли, кроме теоремы 1 , из их предположений вытекает равномерная ограниченность исходной последовательности $\left\{f_{j}\right\}$.

4.5. Для знакопеременной функции $f:[a, b] \rightarrow \mathbb{R}$ обозначим через $\mathscr{P}(f)$ множество всех наборов точек $\left\{t_{1}, \ldots, t_{n}\right\} \subset[a, b]$, где $n \in \mathbb{N}$, таких, что $t_{1}<\cdots<t_{n}$, и либо $(-1)^{i} f\left(t_{i}\right)>0$ для всех $i=1, \ldots, n$, либо $(-1)^{i} f\left(t_{i}\right)<0$ для всех $i=1, \ldots, n$, либо $(-1)^{i} f\left(t_{i}\right)=0$ для всех $i=1, \ldots, n$, и следуя [14], положим

$$
T(f,[a, b])=\sup \left\{\sum_{i=1}^{n}\left|f\left(t_{i}\right)\right| \mid n \in \mathbb{N} \text { и }\left\{t_{i}\right\}_{i=1}^{n} \in \mathscr{P}(f)\right\} ;
$$

если же функция $f$ всюду на $[a, b]$ неотрицательна или всюду на $[a, b]$ неположительна, то полагаем $T(f,[a, b])=\sup _{t \in[a, b]}|f(t)|$. Так определенная величина $T(f,[a, b])$ называется осиилляиией $f$ на $[a, b]$ в смысле Шрадера.

В работе [14; теорема 1.2] установлено следующее обобщение теоремы Хелли: если последовательность функиий $\left\{f_{j}\right\}$ на отрезке $[a, b]$ такова, что

$$
\sup _{j, k \in \mathbb{N}} T\left(f_{j}-f_{k},[a, b]\right)<\infty,
$$

то она содержит подпоследовательность, которая сходится всюду на $[a, b]$. Такая последовательность $\left\{f_{j}\right\}$ является поточечно ограниченной, но никаких свойств 
"регулярности" предельной функции, как в предыдущих обобщениях пп. 4.1-4.4, не утверждается. Приведем примеры, показывающие, что этот результат и теорема 1 независимы (некоторое развитие понятия осцилляции по Шрадеру приведено в работе [15], где установлено соответствующее обобщение теоремы Хелли - теорема 2.1; этот результат и теорема 1 также независимы [16]): последовательность из примера 3.7 удовлетворяет теореме 1, но не удовлетворяет ни одному из приведенных выше обобщений теоремы Хелли, в то время как последовательность $f_{j}(t)=(-1)^{j} \mathscr{D}(t)$ на $[0,1]$ удовлетворяет принципу выбору Шрадера, но не удовлетворяет никакому другому приведенному выше принципу выбора.

4.6. Модуль непрерывности $\omega(\cdot, f):[0, b-a] \rightarrow \mathbb{R}^{+}$непрерывной функции $f:$ $[a, b] \rightarrow \mathbb{R}$ определяется как обычно правилом

$$
\omega(\rho, f)=\sup \{|f(s)-f(t)|: s, t \in[a, b],|s-t| \leqslant \rho\} \quad \text { при } \quad 0<\rho \leqslant b-a
$$

и

$$
\omega(0, f)=\lim _{\rho \rightarrow+0} \omega(\rho, f)=0 .
$$

По теореме Вейерштрасса $\operatorname{osc}(f,[a, b])$ конечна. Последовательность функций $\left\{f_{j}\right\}$ на $[a, b]$ называется равностепенно непрерывной, если

$$
\lim _{\rho \rightarrow+0} \sup _{j \in \mathbb{N}} \omega\left(\rho, f_{j}\right)=0 \text {. }
$$

Имеет место следующая известная теорема Асколи: поточечно ограниченная равностепенно непрерывная последовательность вещественных функций $\left\{f_{j}\right\}$ на $[a, b]$ содержит всюду сходящуюся подпоследовательность. Эта теорема является следствием теоремы 1 , если принять во внимание, что

$$
N\left(\varepsilon, f_{j},[a, b]\right) \leqslant \frac{b-a}{\min \left\{\rho \in \mathbb{R}^{+} \mid \omega\left(\rho, f_{j}\right)=\varepsilon\right\}} \quad \text { для } \quad 0<\varepsilon<\operatorname{osc}\left(f_{j},[a, b]\right) .
$$

Авторы выражают признательность В.С. Балаганскому (Институт математики и механики Уральского отделения РАН, г. Екатеринбург, Россия) и К. Манискалко (C. Maniscalco, Università degli Studi di Palermo, Palermo, Italy) за заинтересованное обсуждение результатов работы и ценные замечания.

\section{СПИСОК ЦИТИРОВАННОЙ ЛИТЕРАТУРЫ}

[1] И. П. Натансон, Теория функиий вещественной переменной, Наука, М., 1974.

[2] R. M. Dudley, R. Norvaiša, Differentiability of Six Operators on Nonsmooth Functions and $p$-Variation, With the collaboration of Jinghua Qian, Lecture Notes in Math., 1703, Springer-Verlag, Berlin, 1999.

[3] S. Gniłka, "On the generalized Helly's theorem", Funct. Approximatio Comment. Math., 4 (1976), 109-112.

[4] J. Musielak, W. Orlicz, "On generalized variations. I", Studia Math., 18 (1959), 11-41.

[5] В.В. Чистяков, "О многозначных отображениях конечной обобщенной вариации", Матем. заметки, 71:4 (2002), 611-632.

[6] V. V. Chistyakov, "Selections of bounded variation", J. Appl. Anal., 10:1 (2004), 1-82. 
[7] V. V. Chistyakov, "A selection principle for functions of a real variable", Atti Semin. Mat. Fis. Univ. Modena Reggio Emilia, 53:1 (2005), 25-43.

[8] M. Schramm, "Functions of $\Phi$-bounded variation and Riemann-Stieltjes integration", Trans. Amer. Math. Soc., 287:1 (1985), 49-63.

[9] D. Waterman, "On $\Lambda$-bounded variation", Studia Math., 57:1 (1976), 33-45.

[10] V. V. Chistyakov, "The optimal form of selection principles for functions of a real variable", J. Math. Anal. Appl., 310:2 (2005), 609-625.

[11] В.В. Чистяков, "Принцип выбора для функций со значениями в равномерном пространстве", Докл. РАН, 409:5 (2006), 591-593.

[12] В. В.Чистяков, "Поточечный принцип выбора для функций одной переменной со значениями в равномерном пространстве", Матем. тр., 9:1 (2006), 176-204.

[13] 3. А. Чантурия, "Модуль изменения функции и его применения в теории рядов Фурье", Докл. АН СССР, 214:1 (1974), 63-66.

[14] K. Schrader, "A generalization of the Helly selection theorem", Bull. Amer. Math. Soc., 78:3 (1972), 415-419.

[15] L. Di Piazza, C. Maniscalco, "Selection theorems, based on generalized variation and oscillation", Rend. Circ. Mat. Palermo (2), 35:3 (1986), 386-396.

[16] C. Maniscalco, "A comparison of three recent selection theorems", Math. Bohem., 132:2 (2007), 177-183.

Ю. В. Третьяченко

Поступило

Нижегородский государственный университет

08.06.2007

им. Н. И. Лобачевского

E-mail: tretyachenko_y_v@mail.ru

\section{В. В. Чистяков}

Государственный университет - Высшая школа экономики

(Нижегородский филиал)

E-mail: czeslaw@mail.ru 